\title{
Efecto de la rehabilitación cardiaca temprana en pacientes incluidos en Código Infarto
}

\author{
Samuel Justiniano-Cordero, ${ }^{1}$ Adrián Tenorio-Terrones, ${ }^{2}$ Gabriela Borrayo-Sánchez, ${ }^{2}$ Raúl Cantero-Colín, ${ }^{2}$ \\ Verónica López-Roldán, ${ }^{3}$ Luis Rafael López-Ocaña, ${ }^{4}$ y José de Jesús Arriaga-Dávila \\ ${ }^{1}$ Programa Nacional de Rehabilitación Cardiaca y Prevención; ${ }^{2}$ Centro Médico Nacional Siglo XXI, Hospital de Cardiología; ${ }^{3}$ División Unidades de \\ Rehabilitación; ${ }^{4}$ Coordinación de Atención Integral en Segundo Nivel; ${ }^{5}$ Dirección de Prestaciones Médicas. Instituto Mexicano del Seguro Social, \\ Ciudad de México, México
}

\begin{abstract}
Resumen
Introducción: La rehabilitación cardiaca temprana (RCT) implementada en el protocolo Código Infarto (Cl) es una estrategia en la atención del infarto agudo de miocardio. El objetivo fue identificar el efecto de la RCT en pacientes incluidos en Cl. Método: Estudio de casos y controles. Se incluyeron pacientes consecutivos con diagnóstico de infarto agudo de miocardio ingresados a un hospital de cardiología entre febrero de 2015 y junio de 2017. Se crearon dos grupos: I y II, antes y después de Cl y RCT. Resultados: Se incluyeron 1141 pacientes: 220 del grupo I y 921 del grupo II, edad $62.64 \pm 10.53$ años; $80.9 \%$ hombres y $19.1 \%$ mujeres. Los principales factores de riesgo para los grupos I y II fueron sedentarismo, 92.7 y $77.8 \%$; dislipidemia, 80.9 y $55.8 \%$; hipertensión, 63.2 y $62 \%$; tabaquismo, 66.8 y $59.2 \%$; y diabetes, 54.5 y $59.1 \%$. En el grupo II se inició antes la rehabilitación $(1.8 \pm 1.6$ y $4.2 \pm 3.2)$ y los días en terapia intensiva y hospitalización fueron menores $(2.4 \pm 2.2$ y $4.8 \pm 4.1 ; 8.6 \pm 5.2$ y $12.3 \pm 7.7$ ), así como los días de incapacidad (58.6 y 67.7). Conclusiones: $C l$ y RCT son estrategias complementarias que permiten alta temprana de terapia intensiva y hospitalización, mejor calidad de vida y menos días de incapacidad laboral.
\end{abstract}

PALABRAS CLAVE: Rehabilitación cardiaca temprana. Código infarto. Calidad de vida.

\begin{abstract}
Introduction: Early cardiac rehabilitation (ECR) implemented in the Infarction Code (IC) protocol is a strategy in the care of acute myocardial infarction. The purpose of this study was to identify the effect of ECR in IC-included patients. Method: Case-control study. Consecutive patients diagnosed with acute myocardial infarction and admitted to a cardiology hospital between February 2015 and June 2017 were included. Two groups were created: I and II, before and after IC and ECR. Results: We included 1141 patients, 220 in group I and 921 in group II, with an age of $62.64 \pm 10.53$ years; $80.9 \%$ were males and $19.1 \%$ females. The main risk factors for groups I and II were sedentariness, $92.7 \%$ versus $77.8 \%$; dyslipidemia, $80.9 \%$ versus $55.8 \%$; hypertension, $63.2 \%$ versus $62 \%$; smoking, $66.8 \%$ versus $59.2 \%$; and diabetes, $54.5 \%$ versus $59.1 \%$. Rehabilitation was started earlier (1.8 \pm 1.6 versus $4.2 \pm 3.2)$ and the days spent in intensive therapy and hospitalization were fewer in group II ( $2.4 \pm 2.2$ versus $4.8 \pm 4.1$ and $8.6 \pm 5.2$ versus $12.3 \pm 7.7, p<0.0001$, respectively), as well as the days of disability (58.6 versus 67.7). Conclusions: IC and ECR are complementary strategies that allow an early discharge from intensive therapy and hospitalization, as well as better quality of life and fewer days of disability leave.
\end{abstract}

KEY WORDS: Early cardiac rehabilitation. Infarction code. Quality of life.

Correspondencia:

Adrián Tenorio-Terrones

E-mail: attdbzgt@hotmail.com
Fecha de recepción: 19-10-2018

Fecha de aceptación: 16-11-2018

DOI: 10.24875/GMM. 18004760
Gac Med Mex. 2019;155:46-51

Disponible en PubMed

www.gacetamedicademexico.com 


\section{Introducción}

En México, las enfermedades cardiovasculares son la primera causa de muerte y una de las principales causas de morbilidad. Afectan cada vez más a hombres y mujeres en edad productiva, ocasionando ausentismo, incapacidad y pensión laboral. En México, el Instituto Nacional de Estadística y Geografía reportó 685766 muertes totales por cualquier causa en 2016, de las cuales aproximadamente 97743 fueron por enfermedades isquémicas del corazón. ${ }^{1-3}$ En el RENASCA (Registro Nacional Síndrome Coronario Agudo) del Instituto Mexicano del Seguro Social (IMSS) se calculó que el costo por el tratamiento inicial del infarto agudo de miocardio con elevación del segmento ST (IAM CEST) era de aproximadamente de 260284 pesos mexicanos, sin considerar los costos a largo plazo. Diversos estudios han demostrado que la rehabilitación cardiaca previene el desarrollo y el avance de la enfermedad cardiaca. ${ }^{4}$

El diagnóstico y tratamiento oportunos en la enfermedad isquémica del corazón son fundamentales e inciden en su evolución, complicaciones y pronóstico a corto y largo plazo. Es fundamental tratar adecuadamente al paciente durante la llamada "hora dorada" para reducir la mortalidad y preservar la función cardiaca: en los pacientes tratados entre las cero y seis horas se registraron $30 / 1000$ muertes comparadas con 20/1000 en los tratados entre las siete y las 12 horas; las diferencias se registraron especialmente en las primeras dos horas. ${ }^{5-8}$

El protocolo de atención de urgencias denominado Código Infarto, del IMSS, permitió mejorar las estrategias de reperfusión y los tiempos de atención en el Hospital de Cardiología del Centro Médico Nacional Siglo XXI, nueve hospitales y dos unidades de medicina familiar, con efectiva comunicación interdisciplinaria y ofreciendo atención 24 horas los siete días a la semana; con este programa se reportaron menos complicaciones tempranas y muertes. ${ }^{9}$

Hace 40 años, los pacientes con IAM CEST eran candidatos a pensión y no se prescribía actividad física. En la actualidad, los programas de rehabilitación cardiaca requieren un equipo multidisciplinario que se conforma por médicos, fisioterapeutas, nutriólogos, psicólogos y enfermeras, entre otros profesionales. ${ }^{10}$ El objetivo de la rehabilitación cardiaca regular es evitar el síndrome de reposo prolongado e iniciar la actividad física después de un evento cardiovascular, el control de los factores de riesgo cardiovascular, atender aspectos psicológicos, así como reintegrar al paciente a su vida social y laboral. ${ }^{11-13}$
El gasto erogado por el IMSS para en el rubro "seguro de enfermedades y maternidad" fue de 329264 millones de pesos en 2017. Desde 2010, las enfermedades cardiovasculares y circulatorias ocupan el primer lugar como causa de años de vida saludable perdidos por muerte prematura y discapacidad, también son el primer lugar como motivo de consulta externa y en 2010 fueron el cuarto lugar en consulta de urgencias. ${ }^{14}$ Por esta razón se han desarrollado programas de rehabilitación cardiaca orientados principalmente a la prevención primaria y secundaria, con beneficios funcionales y en los síntomas del paciente. En México, es reducido el número de centros con programas de rehabilitación cardiaca (menor a $4.4 \%$ ) y a ellos únicamente se designa $6.2 \%$ del producto interno bruto en comparación con $9.3 \%$ promedio en el mundo, registrado por la Organización para la Cooperación y el Desarrollo Económico. ${ }^{15}$

El Hospital de Cardiología del Centro Médico Nacional Siglo XXI del IMSS, desde hace 40 años cuenta con el Servicio de Rehabilitación Cardiaca, cuyo porcentaje de referencia es de $15 \%$. Actualmente, la rehabilitación cardiaca debe iniciarse desde la fase hospitalaria temprana y continuar durante el alta con un programa de ejercicio físico, control de los factores de riesgo y educación respecto a la enfermedad, orientados por un equipo multidisciplinario. La propuesta de nuestro grupo es la rehabilitación cardiaca temprana $(\mathrm{RCT})$, que requiere captación e inicio de la rehabilitación cardiaca desde las primeras 24 horas de la fase I, con seguimiento desde el ingreso a la unidad de cuidados intensivos coronarios (UCIC) y durante dos fases de rehabilitación cardiaca ambulatoria y autoayuda. El protocolo Código Infarto y la RCT son parte del programa "A Todo Corazón" del IMSS para la prevención y atención de enfermedades cardiovasculares. El objetivo del presente trabajo es identificar el efecto de la RTC en pacientes incluidos en Código Infarto, comparado con los resultados obtenidos en pacientes no incluidos en el programa, en quienes se llevó a cabo rehabilitación cardiaca regular.

\section{Método}

Mediante un estudio de casos y controles se incluyeron pacientes de uno y otro sexo, entre 20 y 95 años de edad, con fracción de expulsión mayor a $20 \%$, que ingresaron al Hospital de Cardiología del Centro Médico Nacional Siglo XXI, Ciudad de México, con diagnóstico de IAM CEST de acuerdo con los criterios de la American College of Cardiology, American Heart 
Association y European Society of Cardiology. ${ }^{16}$ El grupo I estuvo integrado por pacientes con diagnóstico de IAM CEST atendidos antes de febrero de 2015, previo a la implementación de Código Infarto, e incluidos en el programa de rehabilitación cardiaca regular. El grupo II incluyó pacientes con diagnóstico de IAM CEST en quienes se siguió el protocolo Código Infarto y se instauró RCT. Se excluyeron pacientes con síndrome coronario agudo sin elevación del segmento ST, estenosis aórtica severa, insuficiencia cardiaca, angina inestable, aneurisma disecante de la aorta, arritmias ventriculares no controladas, embolismo sistémico o pulmonar, enfermedades infecciosas agudas. Todos los pacientes firmaron el consentimiento informado para participar en la rehabilitación cardiaca y en el estudio.

Se utilizó una hoja de captación para analizar los datos obtenidos de los expedientes clínicos. La RCT incluye tres fases:

- Fase I, con inició entre las 24 a 48 horas en la UCIC. Se realizaron movilizaciones y estiramientos, así como deambulación durante la hospitalización (capacidad funcional de 1 a 3.5 Mets).

- Fase II, al alta de la hospitalización, incluye evaluación, estratificación, prescripción de ejercicio físico, educación para la modificación de los factores de riesgo y reintegración a las actividades laborales (capacidad funcional de 3.5 a 10 Mets).

- Fase III, actividad física grupal extrainstitucional (capacidad funcional $>10$ Mets).

Las variables dependientes fueron los días de estancia en la unidad de cuidados intensivos, días totales de hospitalización, días de incapacidad temporal y calidad de vida determinada por el cuestionario de Velasco Del Barrio, para pacientes con patología isquémica y validado al español, que consta de 40 reactivos agrupados en nueve áreas: salud, sueño/ descanso, comportamiento emocional, proyectos de futuro, movilidad, relaciones sociales, comportamiento de alerta, comunicación, ocio/trabajo. ${ }^{17,18}$

El análisis estadístico de las variables cuantitativas se llevó a cabo con la obtención de la tendencia central y dispersión. Para la estadística inferencial se utilizó $\mathrm{t}$ de Student y para las variables cualitativas, chi cuadrada. Se consideró como diferencia significativa una $p<0.05$. Se utilizó el programa estadístico SPSS versión 24 .

\section{Resultados}

Se estudiaron 1141 pacientes, 220 pacientes del grupo I y 921 del grupo II; $80.9 \%$ del sexo masculino
Tabla 1. Características de la población incluida en rehabilitación cardiaca temprana y regular

\begin{tabular}{|c|c|c|c|}
\hline \multirow[t]{2}{*}{ Variable } & $\begin{array}{l}\text { Grupo I } \\
(n=220)\end{array}$ & $\begin{array}{l}\text { Grupo II } \\
(n=921)\end{array}$ & $\mathbf{p}^{+}$ \\
\hline & Media \pm DE & Media \pm DE & \\
\hline Edad (años) & $63 \pm 10.8$ & $63 \pm 10.1$ & NS \\
\hline Peso inicial (kg) & $72.4 \pm 9.7$ & $73.53 \pm 12.2$ & NS \\
\hline Peso final $(\mathrm{kg})$ & $71.8 \pm 9.7$ & $72.29 \pm 11.9$ & NS \\
\hline Cintura inicial (cm) & $96.4 \pm 8.0$ & $96.59 \pm 11.4$ & NS \\
\hline Cintura final (cm) & $95.9 \pm 9.3$ & $93.84 \pm 15.7$ & NS \\
\hline IMC inicial (kg/altura en m²) & $27.1 \pm 3.2$ & $27.34 \pm 3.8$ & NS \\
\hline IMC final (kg/altura en m²) & $26.7 \pm 3.6$ & $26.92 \pm 3.7$ & NS \\
\hline \multirow[t]{2}{*}{ FEV1 $(\%)$} & $46.8 \pm 9.4$ & $46.06 \pm 9.9$ & NS \\
\hline & $\%$ & $\%$ & \\
\hline Diabetes mellitus & 54.5 & 59.1 & NS \\
\hline $\begin{array}{l}\text { Hipertensión arterial } \\
\text { sistémica }\end{array}$ & 63.2 & 62 & NS \\
\hline Tabaquismo & 66.8 & 59.2 & 0.031 \\
\hline Sedentarismo & 92.7 & 77.8 & $<0.0001$ \\
\hline Dislipidemia & 80.9 & 55.8 & $<0.0001$ \\
\hline Obesidad & 19.1 & 27.3 & 0.011 \\
\hline Sobrepeso & 58.6 & 47.9 & 0.025 \\
\hline Depresión & 3.2 & 2.8 & NS \\
\hline \multicolumn{4}{|l|}{$\begin{array}{l}\text { Enfermedad por lesión de } \\
\text { número de vasos }\end{array}$} \\
\hline | & 69 & 47.7 & $<0.0001$ \\
\hline$\|$ & 11.5 & 23.2 & \\
\hline III & 19.5 & 29.1 & \\
\hline \multicolumn{4}{|l|}{$\begin{array}{l}\text { Estratificación de riesgo } \\
\text { según AACVPR }\end{array}$} \\
\hline Bajo & 57.5 & 41.3 & $<0.0001$ \\
\hline Moderado & 15.1 & 24.1 & \\
\hline Alto & 27.4 & 34.6 & \\
\hline
\end{tabular}

Grupo I = sin rehabilitación cardiaca temprana (antes de Código Infarto). Grupo II = con rehabilitación cardiaca temprana (Código Infarto).

+Significación por Chi cuadrada

*t de Student

$\mathrm{DE}=$ desviación estándar, IMC = índice de masa corporal, FEV1 = fracción de expulsión del ventrículo izquierdo, NS = no significativo, $A A C V P R=$ American Association of Cardiovascular and Pulmonary Rehabilitation

y $19.1 \%$ del femenino. La edad promedio del total de los pacientes ingresados fue de $62.6 \pm 10.5$ años. Se registró infarto inferior en $48 \%$, anterior extenso en $33.3 \%$, anteroseptal en $16.8 \%$ y lateral en $2 \%$. Las características antropométricas como peso, índice de masa corporal, medición de cintura inicial y final se muestran en la Tabla 1. Las principales ocupaciones fueron empleado general (44\%) y empleado administrativo $(22.6 \%)$. Los factores de riesgo entre los 
Tabla 2. Impacto de la rehabilitación cardiaca temprana en pacientes con IAM CEST

\begin{tabular}{lccc}
\hline Indicadores & Grupo I $(\mathbf{n}=\mathbf{2 2 0})$ & Grupo II $(\mathbf{n}=\mathbf{9 2 1})$ & $\mathbf{p}$ \\
\hline Días inicio fase I & $4.2 \pm 3.2$ & $1.8 \pm 12.4 \pm 2.2 .6$ & $<0.0001^{*}$ \\
Días estancia UCIC & $4.8 \pm 4.1$ & & $<0.0001^{*}$ \\
Días estancia piso & $7.4 \pm 5.6$ & $6.2 \pm 4.3$ & $0.002^{*}$ \\
Días totales de hospitalización & $12.3 \pm 7.7$ & $8.6 \pm 5.2$ & $<0.0001^{*}$ \\
Días de ITT al alta de fase II & $67.7 \pm 19.1$ & $58.61 \pm 13.4$ & $<0.0001^{*}$ \\
\hline
\end{tabular}

Grupo I = sin rehabilitación cardiaca temprana (antes de Código Infarto).

Grupo II = con rehabilitación cardiaca temprana (Código Infarto).

*t de Student para muestras independientes.

IAM CEST = infarto agudo de miocardio con elevación del segmento ST, UCIC = unidad de cuidados intensivos coronarios, ITT = incapacidad temporal para el trabajo.

grupos de estudio fueron menos frecuentes en el grupo II (Tabla 1).

El tiempo de inicio de la rehabilitación cardiaca fue menor en el grupo II (2.41 días, $p<0.0001)$, así como la estancia en la UCIC (2.46 días, $p<0.0001)$ y el tiempo de hospitalización (1.21 días, $p=0.002$ ). Los días totales de hospitalización en el grupo II disminuyeron (3.64, $\mathrm{p}<0.0001)$; encontramos menos días de incapacidad en el grupo II, 58.6 versus 67.7 días
( $p<0.0001$ ) (Tabla 2). Finalmente, en el grupo II se observó mejoría significativa en el déficit de calidad de vida (cuestionario Velasco Del Barrio) ${ }^{17}$ (Figura 1).

\section{Discusión}

El manejo temprano del enfermo con infarto agudo de miocardio, con intervención en la fase aguda orientada no solo al inicio de la actividad física sino

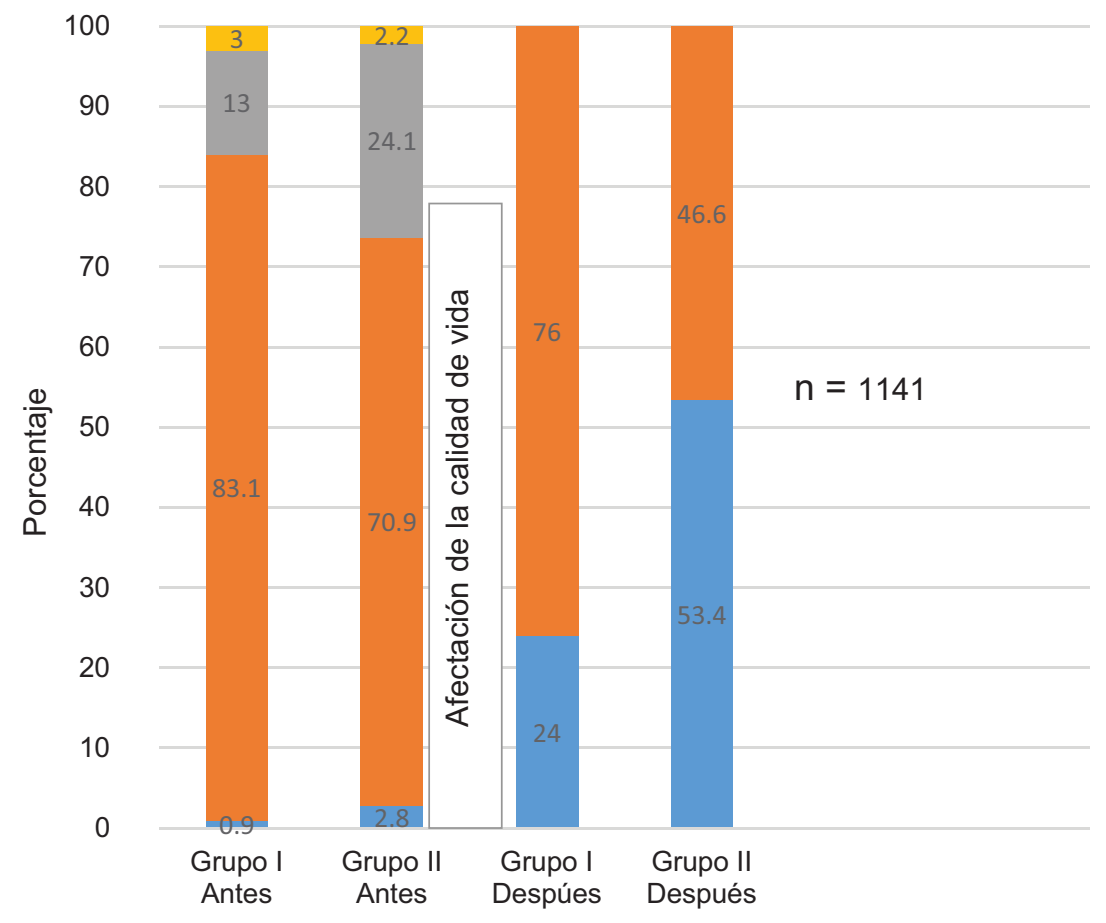

\begin{tabular}{|c|c|c|c|}
\hline Leve & Moderada & Importante & Grave \\
\hline 0 a 50 & 51 a 100 & 101 A 150 & 151 a 200 \\
\hline
\end{tabular}

Figura 1. Calidad de vida según el cuestionario de Velasco Del Barrio. El mayor porcentaje de pacientes con déficit leve y moderado en el grupo II comparado con grupo I, traduce mejoría al final de la rehabilitación cardiaca temprana. 
también a la educación del paciente respecto al conocimiento de su enfermedad y la importancia del control de los factores de riesgo cardiovasculares, logra una más pronta reinserción sociolaboral y reduce los costos por atención y rehospitalización.

Código Infarto es una estrategia reciente en México que incluye el seguimiento en diferentes servicios dentro del proceso de tratamiento intrahospitalario, en adición con técnicas y manejo temprano de la rehabilitación cardiaca, para maximizar el efecto benéfico de las medidas implementadas.

En la población estudiada fue más frecuente el sexo masculino (en ambos grupos) y la edad promedio fue de 62.9 años, similar a los hallazgos de Borrayo et al., ${ }^{9}$ quienes, además, describen una población de mayor riesgo. Al comparar los grupos se observó disminución del peso, cintura e índice de masa corporal en el grupo II al término del programa, si bien fue mínima y no significativa. La supervisión en el gasto energético semanal por paciente para la disminución significativa de peso y así obtener una mejoría sustancial constituye un aspecto por mejorar. ${ }^{19,20}$

Dado que entre los factores que definen la evolución están la fracción de expulsión del ventrículo izquierdo y el número de afección de vasos, identificamos que hubo mayor porcentaje de riesgo alto y moderado en el grupo II. McPhee et al., en un estudio de 486 pacientes, registraron mejoría en la capacidad cardiopulmonar posterior a la RCT antes de 114 días, ${ }^{21}$ con lo que se demuestra que es determinante ofrecer diagnóstico, tratamiento y rehabilitación tempranos para un mejor pronóstico funcional. ${ }^{22}$

La estrategia Código Infarto y la RCT mostraron beneficios importantes. El inicio de la RCT desde urgencias en promedio fue de 1.86 días, con disminución de 2.4 días respecto a la rehabilitación cardiaca regular. Encontramos una reducción de 2.4 días en la estancia en la UCIC y 1.2 días en la hospitalización, lo que sugirió recuperación anticipada y que se evitaron los efectos nocivos de la inmovilidad. ${ }^{21}$ Este beneficio fue resultado de las estrategias de captación y seguimiento de pacientes. ${ }^{23}$ En 2017, Corcoran et al. realizaron un estudio en el que determinaron los efectos de una rehabilitación temprana en UCIC; al igual que en nuestro estudio encontraron disminución significativa de la estancia, de 4.6 a 3.7 días $(p=0.05)$, así como disminución de $40 \%$ en los pacientes con estancia prolongada de 6 a 3.4 días, promedio que observamos en nuestra investigación. ${ }^{24}$

En cuanto a la calidad de vida medida por el cuestionario de Velasco, nuestros resultados fueron positivos, ya que los pacientes del grupo II mostraron mejoría en la calidad de vida, reflejada por un porcentaje mayor de pacientes con déficit leve. Anchah et al. realizaron un estudio de evaluación de calidad de vida en pacientes con síndrome coronario agudo en rehabilitación cardiaca fase I temprana, para lo cual utilizaron el cuestionario SF-36 en un seguimiento de seis a 12 meses; encontraron mejoría en los tratados tempranamente. Nuestros resultados son consecuencia de los diversos programas de atención médica oportuna, programas de educación y entrenamiento físico supervisado..$^{25}$

Además, en nuestro análisis mostramos que la conjunción de Código Infarto y la RCT disminuyeron 9.1 días la incapacidad. Si esta cantidad se extrapola a los diferentes gastos de recursos por costo-día en la atención sanitaria en la UCIC y la hospitalización en segundo y tercer nivel en el IMSS, el posible ahorro por día de hospitalización para el año 2016 pudiera haber sido de 6958 pesos mexicanos. ${ }^{26,27}$ En 1993, Oldridge corroboró los beneficios de costo-efectividad de los programas de rehabilitación cardiaca; posteriormente, Edwards et al., en un metaanálisis de costo-efectividad en rehabilitación cardiaca, que abarcó el periodo de 2000 a 2014, señalaron un promedio de edad y distribución de sexo similar. En nuestro estudio, la edad de presentación mínima fue de 22 años, lo que hizo indispensable el control de los factores de riesgo cardiovascular. ${ }^{28}$ También Corcoran et al. señalaron que existe ahorro significativo al realizar rehabilitación temprana; indicaron un ahorro neto de 1.5 millones de dólares. ${ }^{24}$

En nuestro estudio obtuvimos resultados positivos de acuerdo con la referencia a fase II de rehabilitación cardiaca: en el Hospital de Cardiología previamente se tenía $15 \%$ de referencia y actualmente es de $89 \%$ con la implementación de los siete ejes del programa "A todo corazón" del IMSS, que incluye Código Infarto y la RCT, sin embargo, aún es necesario aumentar esta referencia en los diversos centros donde se atiende cardiopatía isquémica, para obtener beneficios sustanciales de la RCT. Entre los diversas factores involucrados en la referencia a rehabilitación cardiaca, Goel et al. señalan el papel del cardiólogo clínico o médico tratante; de igual forma, señalan el aumento de la referencia a rehabilitación cardiaca en los últimos 10 años. ${ }^{22}$

Las limitaciones de nuestro estudio estribaron en el reducido número de pacientes control y el seguimiento respectivo de la población, aspectos que deberán mejorarse en futuras investigaciones y en la evaluación pertinente de la disminución del riesgo cardiovascular. 


\section{Conclusiones}

Identificamos efecto favorable de la implementación de la RCT en los pacientes con infarto agudo de miocardio incluidos en el programa Código Infarto.

Derivado de un conjunto de acciones de mejora en el diagnóstico y tratamiento oportunos y la rehabilitación temprana, se obtuvo una mejor captación y cumplimiento del programa en la fase I, reducción significativa de los días de estancia en UCIC, hospitalización e incapacidad y mejoría en la calidad de vida. Estos resultados indican una mejor calidad en la atención de los pacientes con infarto agudo de miocardio, por lo que se sugiere un modelo de RCT replicable en todas las unidades con la estrategia Código Infarto.

\section{Bibliografía}

1. Plan Nacional de Desarrollo 2013-2018. México: Diario Oficial de la Federación 2013 May 20.

2. Gutiérrez JP, Rivera-Dommarco J, Shamah-Levy T, Villalpando-Hernández S, Franco A, Cuevas-Nasu L, et al. Encuesta Nacional de Salud y Nutrición 2012. México: Instituto Nacional de Salud Pública; Secretaría de Salud; 2012

3. Instituto Nacional de Estadística, Geografía e Informática. [Sitio web]. Mortalidad 2016. México: INEGl; 2016. Disponible en: https://www.inegi. org. $\mathrm{mx} /$ programas/mortalidad

4. Borrayo-Sánchez G, Madrid-Miller A, Arriaga-Nava R, Ramos-Corrales MA, García-Aguilar J, Almeida-Gutiérrez E. Riesgo estratificado de los síndromes coronarios agudos. Resultados del primer RENASCA-IMSS. Rev Med Inst Mex Seguro Soc. 2010;48:259-264.

5. Chiu S, Lupercio K, Nava JR. Manual de conducta y terapéutica cardiovascular. México: Manual Moderno; 2014.

6. O'Gara PT, Kushner FG, Ascheim DD, Casey DE, Chung MK, De Lemos JA, et al. 2013 ACCF/AHA guideline for the management of ST-elevation myocardial infarction: a report of the American College of Cardiology Foundation/American Heart Association Task Force on Practice Guidelines. Circulation. 2013;127:e362-e425.

7. Steg PG, James SK, Atar D, Badano LP, Blömstrom-Lundqvist C Borger MA, et al. ESC Guidelines for the management of acute myocardial infarction in patients presenting with ST-segment elevation. Eur Heart J. 2012;33:2569-2619.

8. Larios, R. Factores de riesgos asociados con infarto agudo al miocardio en derechohabientes atendidos en el H.G.R. No.1 Vicente Guerrero, Acapulco, Guerrero. [Tesis de especialidad]. México: Instituto Politécnico Nacional; 2011.

9. Borrayo-Sánchez G, Álvarez-Covarrubias H, Pérez-Rodríguez G, Arizmendi-Uribe E, Ramírez-Arias E, Peralta-Rosas M, et al. Impacto en la implementación de Código Infarto en pacientes con infarto agudo de miocardio con elevación del segmento ST en el Hospital de Cardiología en el Centro Médico Nacional Siglo XXI. Gac Med Mex. 2017;153:S13-S17.
10. Martínez-Sánchez $\mathrm{C}$, Arias M, González H. Síndromes isquémicos coronarios agudos. México: Intersistemas; 2010.

11. Brown RA. Rehabilitation of patients with cardiovascular diseases. Report of a WHO Expert Committee. World Health Organ Tech Rep Ser. 1964;270:3-46.

12. Ilarraza-Lomelí $\mathrm{H}$, García-Saldivia M, Rojano-Castillo J, Justiniano S, Cerón N, Aranda-Ayala Z, et al. National Registry of Cardiac Rehabilitation Programs in Mexico II (RENAPREC II). Arch Cardiol Mex. 2017;87:270-277.

13. Cortés-Bergoderi $M$, López-Jíménez $F$, Herdy $A H$, Zeballos $C$, Anchique C, Santibáñez C, et al. Availability and characteristics of cardiovascular rehabilitation programs in South America. J Cardiopulm Rehabil Prev. 2013;33:33-34.

14. Informe al Ejecutivo Federal y al Congreso de la Unión sobre la situación financiera y los riesgos del Instituto Mexicano del Seguro Social 20162017. México: Instituto Mexicano del Seguro Social; 2018.

15. Organización para la Cooperación y Desarrollo Económico. [Sitio web]. Indicators 2014. OECD.

16. Ibáñez $B$, James $S$, Agewall $S$, Antunes MJ, Bucciarelli-Ducci $C$, Bueno $\mathrm{H}$, et al. Guía ESC 2017 sobre el tratamiento del infarto agudo de miocardio en pacientes con elevación del segmento ST. Rev Esp Cardiol. 2017;70:1082.e1-e61.

17. Velasco J, Del Barrio V, Mestre MV, Penas C, Ridocci F. Validación de un nuevo cuestionario para evaluar la calidad de vida en pacientes posinfarto. Rev Esp Cardiol. 1993;46:552-558.

18. Soto M, Failde I. Health-related quality of life as an outcome measure in patients with ischemic cardiopathy. Rev Soc Esp Dolor. 2004;11:505-514.

19. Squires R, Kaminsky L, Porcari J, Ruff J, Savage P, Williams M. Progression of exercise training in early outpatient cardiac rehabilitation: an official statement from the American Association of Cardiovascular And Pulmonary Rehabilitation. J Cardiopulm Rehabil Prev. 2018;38:139-146.

20. Hansen D, Niebauer J, Cornelissen V, Barna O, Neunhäuserer D, Stettler $\mathrm{C}$, et al. Exercise prescription in patients with different combinations of cardiovascular disease risk factors: a consensus statement from the EXPERT Working Group. Sports Med. 2018;48:1781-1797.

21. McPhee PG, Winegard KJ, McDonald MJ, McKelvie RS, Millar P. Importance of early cardiac rehabilitation on changes in exercise capacity: a retrospective pilot study. Appl Physiol Nutr Metab. 2015;40:1314-1317.

22. Goel K, Lennon R, Tilbury $R$, Squires $R$, Thomas $R$. Impact of cardiac rehabilitation on mortality and cardiovascular events after percutaneous coronary intervention in the community. Circulation 2011;123:2344-2352.

23. Oldridge N, Furlong W, Feeny D, Torrance G, Guyatt G, Crowe J, et al. Economic evaluation of cardiac rehabilitation soon after acute myocardial infarction. Am J Cardiol. 1993;72:154-161.

24. Corcoran JR, Herbsman JM, Bushnik T, Van-Lew S, Stolfi A, Parkin K, et al. Early rehabilitation in the medical and surgical intensive care units for patients with and without mechanical ventilation: an Interprofessional Performance Improvement Project. PMR. 2017;9:113-119.

25. Anchah, L, Azmi M, Slaw M, Ibrahim M, Sim K, Ong T. Health related quality of life assessment in acute coronary syndrome patients: the effectiveness of early phase I cardiac rehabilitation. Health Qual Life Outcomes. 2017;15:10.

26. Acuerdo ACDO. AS3.HCT.270116/8.P.DF dictado por el H. Consejo Técnico en la sesión ordinaria celebrada el día 27 de enero de dos mil dieciséis, relativo a la aprobación de los costos unitarios por Nivel de Atención Médica para el ejercicio fiscal. México: Diario Oficial de la Federación; 2016.

27. Instituto Mexicano del Seguro Social. Información ejecutiva de la Dirección de Información en Salud. 20 principales motivos de egresos hospitalarios 2013, camas censables. México: IMSS; 2014

28. Edwards $\mathrm{K}$, Jones $\mathrm{N}$, Newton J, et al. The cost-effectiveness of exercise-based cardiac rehabilitation: a systematic review of the characteristics and methodological quality of published literature. Health Econ Rev. 2017:19:37. 